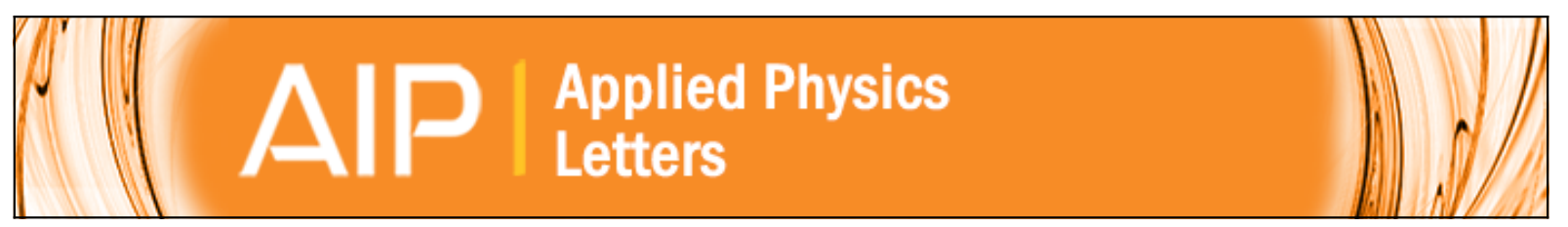

Temperature and Co thickness dependent sign change of the anomalous Hall effect in Co/Pd multilayers: An experimental and theoretical study

V. Keskin, B. Akta, J. Schmalhorst, G. Reiss, H. Zhang, J. Weischenberg, and Y. Mokrousov

Citation: Applied Physics Letters 102, 022416 (2013); doi: 10.1063/1.4776737

View online: http://dx.doi.org/10.1063/1.4776737

View Table of Contents: http://scitation.aip.org/content/aip/journal/apl/102/2?ver=pdfcov

Published by the AIP Publishing 


\title{
Temperature and Co thickness dependent sign change of the anomalous Hall effect in Co/Pd multilayers: An experimental and theoretical study
}

\author{
V. Keskin, ${ }^{1,2}$ B. Aktaş, ${ }^{2}$ J. Schmalhorst,,${ }^{1, a)}$ G. Reiss, ${ }^{1}$ H. Zhang, ${ }^{3}$ J. Weischenberg, ${ }^{3}$ \\ and Y. Mokrousov ${ }^{3}$ \\ ${ }^{1}$ Thin Films and Nanostructures, Department of Physics, Bielefeld University, D-33501 Bielefeld, Germany \\ ${ }^{2}$ Physics Department, Gebze Institute of Technology, Gebze, 41400 Kocaeli, Turkey \\ ${ }^{3}$ Peter Grünberg Institute and Institute for Advanced Simulation, Forschungszentrum \\ Jülich and Jülich-Aachen Research Alliance, D-52425 Jülich, Germany
}

(Received 5 December 2012; accepted 3 January 2013; published online 16 January 2013)

\begin{abstract}
The anomalous Hall effect in ultra-thin $\left\{\mathrm{Co}^{0.3 \mathrm{~nm}} / \mathrm{Pd}^{0.5 \mathrm{~nm}}\right\}_{\mathrm{n}}$ multilayers has been investigated recently with respect to surface and interface contributions [Guo et al., Phys. Rev. B 86, 104433 (2012)]. In this work, we observe a Co thickness and temperature dependent sign change also for

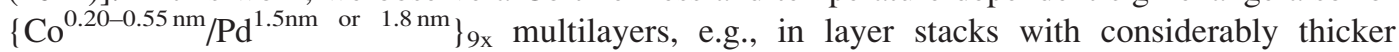
Pd layers and hence lower resistivity. The thickness dependent behavior can be reproduced by $a b$ initio calculations of the Hall conductivity, for which only interfacial and bulk contributions play a role. (C) 2013 American Institute of Physics. [http://dx.doi.org/10.1063/1.4776737]
\end{abstract}

The anomalous Hall effect (AHE) $)^{1,2}$ of ultra-thin multilayered (ML) films with large perpendicular magnetic anisotropy (PMA) has recently attracted considerable attention due to its potential applications for high-density magnetic recording technology, such as recording media, magnetic random access memory (MRAM), and magnetic sensors. ${ }^{3-7}$

For applications, parameters like squareness and coercivity of the magnetic hysteresis curve and the amplitude of the AHE voltage are desired to be tunable. For instance, the coercivity has to be as small as possible when it is used for magnetic field sensor elements, but it has to be moderately large when it is used for recording media elements.

The empirical expression of the Hall resistivity is given as $\rho_{x y}=\rho_{O H}+\rho_{A H E}=R_{0} \cdot \mu_{0} H+R_{S} M$ (Refs. 8 and 9), where $\rho_{O H}=R_{O} \cdot \mu_{0} H$ represents the ordinary Hall resistivity $\left(\mathrm{R}_{0}\right.$ is the ordinary Hall coefficient). The anomalous Hall resistivity $\rho_{A H E}=R_{S} \cdot M$ is obtained by subtracting a straight line $R_{0} \mu_{0} H$ from the Hall resistivity $\rho_{x y}$ measured in dependence of $\mathrm{H}$. In this work, the ordinary contribution is small compared to $\rho_{A H E}$ for all samples.

Since $\mathrm{Co} / \mathrm{Pd}$ is one of the promising candidates for perpendicular magnetic recording technology, ${ }^{3-5}$ there are several AHE studies on thin Co-Pd alloys and $\{\mathrm{Co} / \mathrm{Pd}\}_{\mathrm{n}}$ multilayers. In chronological order, Kim et al. ${ }^{10}$ explored the Pd thickness dependence of the AHE of $\{\mathrm{Co} / \mathrm{Pd}\}$ multilayers prepared by thermal evaporation at room temperature and observed a polarity change of the AHE between 4 and 5 monolayers of Pd for a fixed Co thickness of one monolayer. It was speculated that this can be attributed to a change of the position of the Fermi level with increasing Pd thickness. Jen et al., ${ }^{11}$ studied the AHE coefficient $\left(\mathrm{R}_{\mathrm{S}}\right)$ of $\mathrm{Co}_{1-\mathrm{x}} \mathrm{Pd}_{\mathrm{x}}$ alloys. With increasing $\mathrm{Pd}$ concentration $\mathrm{x}$, they observed a sign change from positive to negative at $\mathrm{x}=0.77$ and claimed that the AHE is dominated by the side jump mechanism for $\mathrm{x} \leq 0.65$, whereas for $\mathrm{x}>0.65$, both the side jump and skew scattering mechanism are equally effective.

\footnotetext{
${ }^{\text {a) }}$ Author to whom correspondence should be addressed: Electronic mail: jan.schmalhorst@uni-bielefeld.de.
}

Aoki et al. ${ }^{12}$ studied $\{\mathrm{Co} / \mathrm{Pd}\}$ multilayers deposited from e-gun sources at room temperature. For a constant $\mathrm{Pd}$ thickness of $2.0 \mathrm{~nm}$, they found that at $4.2 \mathrm{~K}$, the sign of $R_{S}$ changes for a Co layer thickness of about $\mathrm{d}_{\mathrm{Co}}=0.2 \mathrm{~nm}$ compared to $\mathrm{d}_{\mathrm{Co}}=1.6 \mathrm{~nm}$. Stimulated by the work of Jen et al., they attributed their observation to an interfacial alloying effect. However, they mentioned that they could not rule out the possibility that the sign change was a direct result of the different easy axes orientation for $\mathrm{d}_{\mathrm{Co}}=0.2 \mathrm{~nm}$ (showing perpendicular magnetic anisotropy) and $\mathrm{d}_{\mathrm{Co}}=1.6 \mathrm{~nm}$ (inplane anisotropy).

Recent studies on $\{\mathrm{Co} / \mathrm{Pd}\}$ multilayers observed a reversal of the AHE polarity both with repetition number ${ }^{1,13}$ and aging effects at room temperature ${ }^{13}$ in the limit of small Co thickness $(0.2 \mathrm{~nm}$ in Ref. 13 and $0.3 \mathrm{~nm}$ in Ref. 1). This was attributed to a decreasing importance of the surface scattering with increasing number of multilayers compared to the bulk and interface scattering, respectively.

In this work, we report a Co thickness and temperature

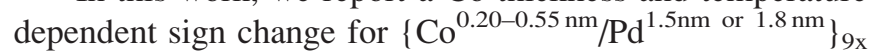
multilayers showing a high PMA with nearly perfect rectangular magnetization switching in the out-of-plane direction and compare the experimental results with $a b$ initio calculations of the anomalous Hall conductivity (AHC).

The $\{\mathrm{Co} / \mathrm{Pd}\}_{9 \mathrm{x}}$ multilayers with Co thickness ranging from $0.20 \mathrm{~nm}$ to $0.55 \mathrm{~nm}$ by $0.05 \mathrm{~nm}$ steps and a fixed $\mathrm{Pd}$ thickness of $1.5 \mathrm{~nm}$ or $1.8 \mathrm{~nm}$ were grown on thermally oxidized $\mathrm{Si}$ wafers by using dc magnetron sputtering. A $5 \mathrm{~nm}$ thick Ta buffer layer was deposited prior to the multilayers. The stacks were covered by a $2.1 \mathrm{~nm}$ thick $\mathrm{MgO}$ layer to prevent oxidation. The samples were annealed at $200^{\circ} \mathrm{C}$ for $60 \mathrm{~min}$ in a magnetic field of 6500 Oe perpendicular to the film plane to enhance the perpendicular magnetic anisotropy of the $\{\mathrm{Co} / \mathrm{Pd}\}$ multilayers. To fabricate the Hall bars, the films were patterned by UV lithography and Ar-ion beam etching to an effective length of $\mathrm{L}=1.2 \mathrm{~mm}$ and a width of $\mathrm{w}=1.0 \mathrm{~mm}$. The Hall voltage $\mathrm{V}_{\mathrm{H}}$ (measured in y-direction) and the longitudinal voltage in $\mathrm{x}$-direction $\left(\mathrm{V}_{\mathrm{x}}\right)$ were driven by a bias current in the $\mathrm{x}$-direction $\left(\mathrm{I}_{\mathrm{x}}=500 \mu \mathrm{A}\right)$, the 
magnetic field $\mathrm{H}$ of up to $10 \mathrm{kOe}$ was applied perpendicular to the film plane in $\mathrm{z}$-direction. The low-temperature AHE measurements were done in a closed-cycle helium cryostat (Oxford Cryodrive 1.5) with a temperature range of $15-330 \mathrm{~K}$. The amplitude of the longitudinal magneto resistance $R_{x x}(H)=V_{x}(H) / I_{x}$ was observed to be less than $0.5 \%$ and will not be further discussed here. The longitudinal resistivity is defined as $\rho_{x x}=\left(V_{x} / I_{x}\right)(w / L) t$, the Hall resistivity accordingly $\rho_{x y}=\left(V_{H} / I_{x}\right) t$, where $t$ is the total thickness of the metallic material including $5 \mathrm{~nm}$ of Ta.

Sputtered Cobalt-Palladium multilayers have an artificial superlattice structure, where Co and Pd grow textured in the (111) direction and where a compositionally sharp interface can be fabricated. ${ }^{14}$ We have confirmed the (111) texture in our films by $\mathrm{x}$-ray diffraction, whereas the in-plane orientation of the grains was random. The according lattice spacings of our films in the growth direction fit very well with the data reported in Ref. 14: $0.2236 \mathrm{~nm}$ for $\mathrm{d}_{\mathrm{Co}}=0.20 \mathrm{~nm}, 0.2226 \mathrm{~nm}$ for $\mathrm{d}_{\mathrm{Co}}=0.30 \mathrm{~nm}$, and $0.2209 \mathrm{~nm}$ for $\mathrm{d}_{\mathrm{Co}}=0.55 \mathrm{~nm} \quad(\mathrm{Pd}$ thickness was $1.5 \mathrm{~nm}$ in all cases). Furthermore, the Pd atoms at the interface to the Co layer can be expected to be magnetically polarized by the Co atoms. ${ }^{14}$

The thickness dependent room temperature (RT) data of the Hall resistivity $\rho_{\mathrm{H}}$ as a function of the out-of-plane magnetic field $\mathrm{H}$ are plotted in Fig. 1 for $\mathrm{d}_{\mathrm{Co}}=0.20,0.25,0.30$, and $0.55 \mathrm{~nm}$. It is obvious that the polarity of the AHE loops remains negative for the thin films $(0.20 \mathrm{~nm}$ and $0.25 \mathrm{~nm} \mathrm{Co})$ and that it changes to positive for the thicker ones $(0.30 \mathrm{~nm}$ to $0.55 \mathrm{~nm} \mathrm{Co}$ ). This behavior is similar to the data reported by Aoki et al. ${ }^{12}$ However, in our case, the sign change is also observed at room temperature and-because of the high outof-plane remanence for all multilayers_-it can be excluded that the sign change results from a rotation of the easy magnetic axis from out-of-plane to in-plane. The coercive field $\mathrm{H}_{\mathrm{C}}$ increases from about $100 \mathrm{Oe}$ for the thinnest film up to 700 Oe $(0.30 \mathrm{~nm} \mathrm{Co})$ and dropped to 120 Oe with increasing Co thickness, as displayed in the inset of Fig. 1.

The critical (Curie-) temperature $\mathrm{T}_{\mathrm{C}}$ for the magnetic phase transition of the $\left\{\mathrm{Co}^{0.20 \mathrm{~nm}} / \mathrm{Pd}^{1.8 \mathrm{~nm}}\right\}_{9 \mathrm{x}}$ multilayer is

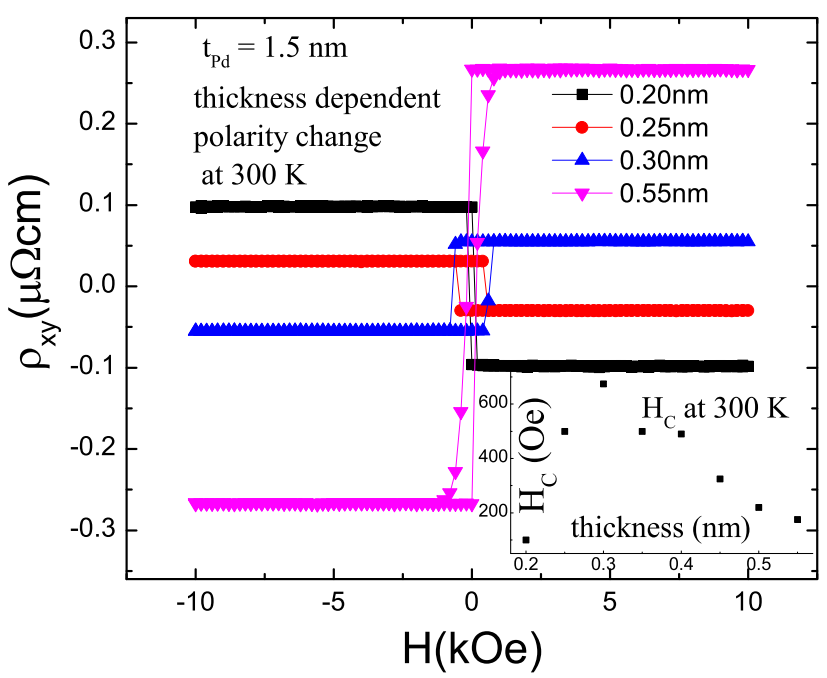

FIG. 1. (a) Hall resistivity as a function of magnetic field for samples of $0.20,0.25,0.30$, and of $0.55 \mathrm{~nm}$ of $\mathrm{Co}$ at RT. Inset: The coercivity field $\mathrm{H}_{\mathrm{C}}$ of all samples at RT. The Pd thickness was $1.5 \mathrm{~nm}$. observed at about $300 \mathrm{~K}$ as can be seen in Fig. 2(b), however, the AHE resistivity is negative for all temperatures. In contrast for the $\left\{\mathrm{Co}^{0.35 \mathrm{~nm}} / \mathrm{Pd}^{1.8 \mathrm{~nm}}\right\}_{9 \mathrm{x}}$ multilayer $\rho_{\mathrm{xy}}$ exhibits a temperature dependent sign change between $140 \mathrm{~K}$ and $160 \mathrm{~K}$ from negative at lower temperatures to positive at higher temperatures. This temperature dependent polarity change is also observed for the $\left\{\mathrm{Co}^{0.30 \mathrm{~nm}} / \mathrm{Pd}^{1.5 \mathrm{~nm}}\right\}_{9 \mathrm{x}}$ multilayer with a transition temperature between $120 \mathrm{~K}$ and $140 \mathrm{~K}$ (see, Figs. 2(a) and 3(b)). However, the nearly perfect squareness of the hysteresis loops is conserved in all measurements, and the normal reduction of $\mathrm{H}_{\mathrm{C}}$ with increasing temperature is also observed for all samples.

Similar trends have been observed both for multilayers with $1.8 \mathrm{~nm}$ and $1.5 \mathrm{~nm}$ Pd (see, Fig. 3(b)), although the critical temperature of the sample with $\mathrm{d}_{\mathrm{Co}}=0.20 \mathrm{~nm}$ and $1.5 \mathrm{~nm}$ $\mathrm{Pd}$ is also above $330 \mathrm{~K}$ and, therefore, larger than in the case of $\mathrm{d}_{\mathrm{Pd}}=1.8 \mathrm{~nm}$ (see, Fig. 1). For this sample, the Hall resistivity $\rho_{\text {xy }}$ exhibits a non-monotonic temperature dependence with a broad local minimum around $140 \mathrm{~K}$ (see, Fig. 3(b)). For larger Co thickness, a monotonically increasing $\rho_{\mathrm{xy}}$ was observed.

In general, the longitudinal resistivity $\rho_{\mathrm{xx}}$ increases monotonically with temperature and with a reduction of the film thickness, which is a well known phenomenon for metal thin films ${ }^{15}$ and multilayers ${ }^{16}$ (see, Fig. 3(a)): the influence of the interface scattering becomes more important if the

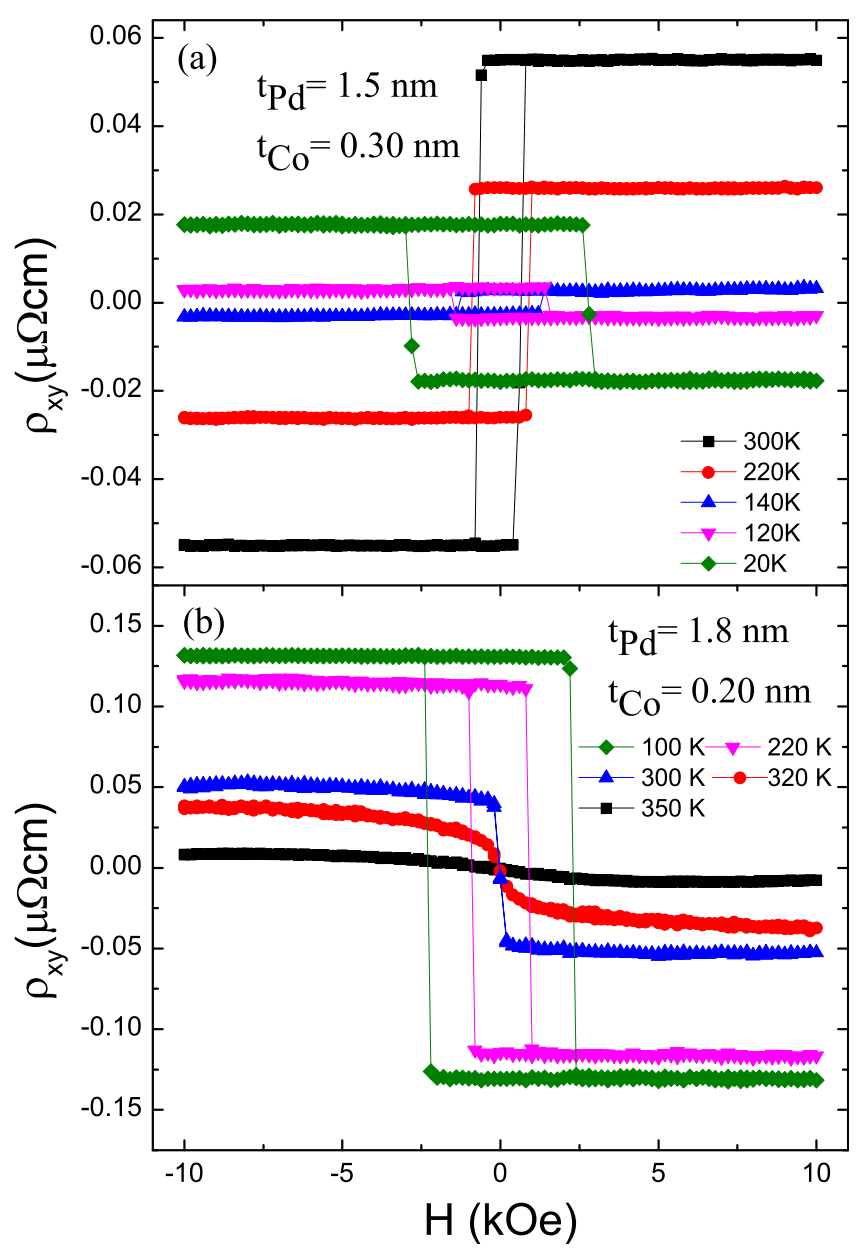

FIG. 2. $\rho_{\mathrm{xy}}$ vs. magnetic field $\mathrm{H}$ at different temperatures $\mathrm{T}$ for (a) $\mathrm{d}_{\mathrm{Co}}$ $=0.30 \mathrm{~nm}$ (the Pd thickness was $1.5 \mathrm{~nm}$ ) and (b) $\mathrm{d}_{\mathrm{Co}}=0.20 \mathrm{~nm}$ (the $\mathrm{Pd}$ thickness was $1.8 \mathrm{~nm}$ ). 


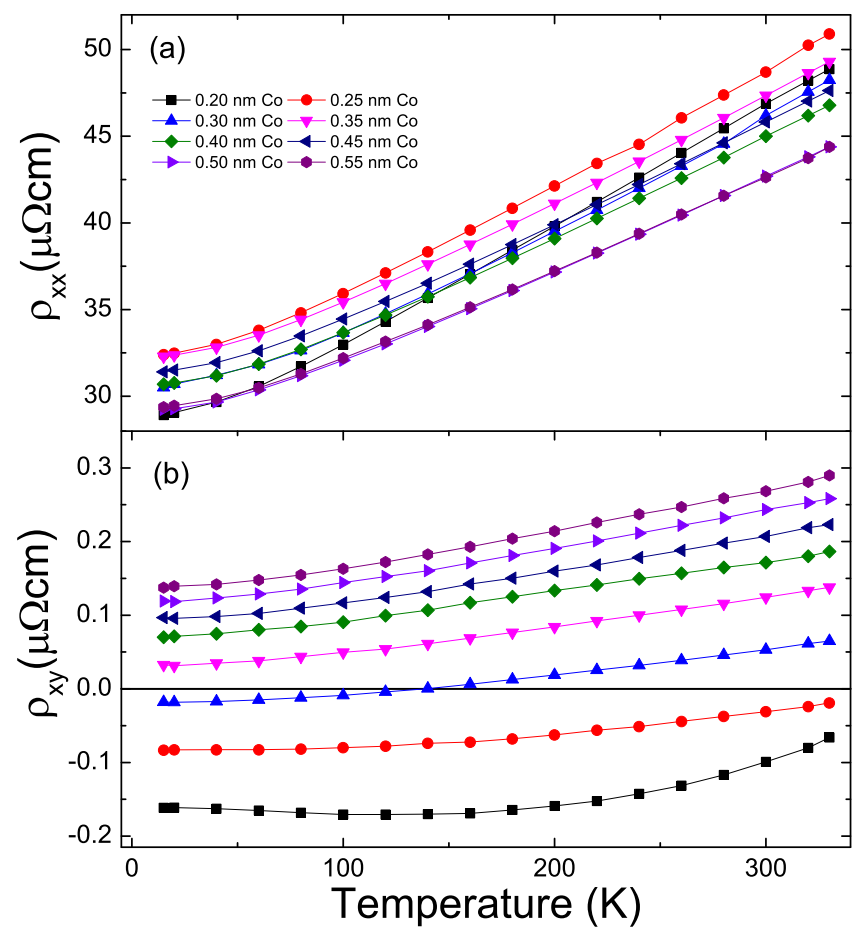

FIG. 3. Temperature dependence of (a) $\rho_{\mathrm{xx}}$ and (b) $\rho_{\mathrm{xy}}$ for all samples with Pd thickness of $1.5 \mathrm{~nm}$.

number of repetitions and, accordingly, the number of interfaces in the multilayer remains constant while the thickness of one or both single layers is reduced (here the Co thickness is reduced). This is an important hint to a significant influence of the $\mathrm{Co} / \mathrm{Pd}$ interface on the electrical properties of the multilayers. One, therefore, should also expect a significant effect on the Hall resistivity.

Conventionally, the dependence of the Hall resistivity $\rho_{x y}$ is plotted vs. the longitudinal resistivity $\rho_{x x}$ to distinguish between intrinsic, side-jump and skew scattering contributions. ${ }^{17}$ However, in the case of multilayers, this is not always helpful for understanding the physical origin of the temperature dependence of $\rho_{x y}$ because of bulk, interface and surface contributions to both, $\rho_{x x}$ and $\rho_{x y}$, and an accordingly inhomogeneous current distribution in the stack. ${ }^{16}$ Furthermore, all contribution might have different temperature dependencies. But one can only measure two voltages $\left(\mathrm{V}_{\mathrm{x}}\right.$ and $\left.\mathrm{V}_{\mathrm{H}}\right)$ and, therefore, a non-ambiguous separation between all contributions can be hardly done.

Therefore, we have chosen a different approach to understand the experimental low temperature data, where thermal excitations are suppressed. We performed first principles calculations of the electronic properties of $\{\mathrm{Co} / \mathrm{Pd}\}$ multilayers with various number of $\mathrm{Co}(\mathrm{Pd})$ atomic layers using the fullpotential linearized augmented plane wave (FLAPW) code FLEUR $^{18}$ and the generalized gradient approximation for the exchange correlation potential. ${ }^{19}$ A total number of 288 kpoints in the Brillouin zone (BZ) was used to do self-consistent calculations with spin-orbit coupling. The supercells to simulate the multilayers were constructed assuming fcc stacking of all atomic layers along (111) direction with constant in-plane lattice constants derived from the experimental lattice constant of Pd $(3.89 \AA)$. The distance between Co atomic layers $(1.7 \AA)$ was taken to be the distance between Co layers as in bulk hcp
Co (reduced because of the constant volume approximation), and the distance between Co and Pd layers was taken to be the average of those of Co and Pd layers. The intrinsic AHC is calculated using the Wannier interpolation technique, ${ }^{20-22}$ given by the Kubo formula

$$
\begin{gathered}
\sigma_{i j}=-e^{2} \hbar \int_{B Z} \frac{d^{3} k}{8 \pi^{3}} \Omega_{i j}(\mathbf{k}), \\
\Omega_{i j}(\mathbf{k})=-2 \operatorname{Im} \sum_{\mathrm{n}, \mathrm{m}}^{\mathrm{o}, \mathrm{e}} \frac{\left\langle\psi_{\mathrm{nk}}\left|\mathbf{v}_{\mathrm{i}}\right| \psi_{\mathrm{m} \mathbf{k}}\right\rangle\left\langle\psi_{\mathrm{m} \mathbf{k}}\left|\mathbf{v}_{\mathrm{j}}\right| \psi_{\mathrm{nk}}\right\rangle}{\left(\varepsilon_{\mathrm{nk}}-\varepsilon_{\mathrm{mk}}\right)^{2}},
\end{gathered}
$$

which relates the conductivity tensor $\sigma_{i j}$ (Eq. (1)) to the BZ integral of the k-dependent Berry curvature tensor $\Omega_{i j}$ (Eq. (2)). In the latter expression, $\psi_{\mathrm{nk}}$ and $\psi_{\mathrm{mk}}$ are, respectively, the occupied (o) and empty (e) eigenstates, $\varepsilon_{\mathrm{nk}}$ and $\varepsilon_{\mathrm{mk}}$ are their eigenenergies, and $v_{i}$ and $v_{j}$ are the Cartesian components of the velocity operator $\mathbf{v}$.

Fig. 4 displays the $\mathrm{AHC}$ of $\{\mathrm{Co} / \mathrm{Pd}\}$ multilayers with various composition calculated using the first principles methods. We considered here the intrinsic contribution given by the Kubo formula above. Obviously, the AHC varies significantly with respect to the location of the Fermi energy $E_{F}$ due to the fact that the Berry curvature $\Omega$ is a sensitive quantity. For instance, the $\mathrm{AHC}$ of $\mathrm{Co}_{3} \mathrm{Pd}_{6}$ multilayers oscillates between positive and negative values. This can cause a nontrivial temperature dependence of the intrinsic AHC. However, to compare with experimental observations at low temperature, the $\mathrm{AHC}$ around $\mathrm{E}_{\mathrm{F}}$ is relevant. As shown in the inset of Fig. 4, the $\mathrm{AHC}$ at $\mathrm{E}_{\mathrm{F}}$ has a strong dependence to the number of Co atomic layers. The sign of AHC is changed from negative to positive for multilayers with more than 2 Co layers $(0.34 \mathrm{~nm})$. This is in agreement with our experimental results that at low temperature, the sign of $\rho_{x y}$ is changed at a critical thickness of $0.30 \sim 0.35 \mathrm{~nm}$ (see, Fig. 3(b)).

In order to roughly estimate the magnitude of the extrinsic contribution to the AHE in the system, we additionally

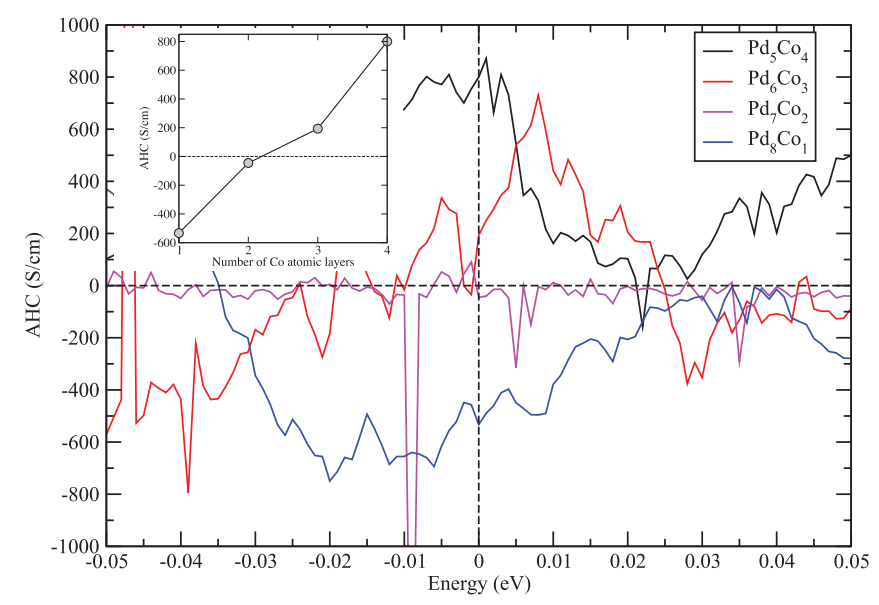

FIG. 4. Anomalous Hall conductivities of $\mathrm{Co}_{\mathrm{m}} \mathrm{Pd}_{\mathrm{n}}$ multilayers. Compositions with $\mathrm{m}=1,2,3,4$ and corresponding $\mathrm{n}=8,7,6,5$ are considered with 9 atomic layers in total to maintain fcc stacking. Horizontal (vertical) dashed line indicates the zero AHC (location of the Fermi energy). The inset displays the dependence of the AHC at the Fermi energy $E_{F}$ with respect to the number of Co atomic layers. The solid line is the guide for the eyes. 
performed an ab intio assessment of the side-jump contribution, which can have a comparatively large magnitude in transition-metals, see, e.g., Refs. 23-25. Since the exact details of disorder in the system are unknown and the skew-scattering contribution to the AHE becomes increasingly suppressed as the temperature of the sample is increased in transition-metals,${ }^{17}$ we choose a model of Gaussian disorder to estimate the so-called scattering-independent side-jump contribution, known to be important in such metals as $\mathrm{Fe}, \mathrm{Co}$, and FePd. ${ }^{25}$ Our zero-temperature calculations performed according to the methodology of Ref. 25 show that the magnitude of the sidejump in $\mathrm{Co} / \mathrm{Pd}$ multilayers does not exceed the magnitude of the intrinsic contribution and has the same sign as the intrinsic values. Noticably, while for the $\mathrm{Co}_{1} \mathrm{Pd}_{8}$ multilayer, the values of both side-jump and intrinsic contributions are negative, they converge to the bulk Co values ${ }^{25}$ as the number of the Co layers in the system is increasing. The overall trend with increasing Co thickness, which is in agreement with experimental observations, provides a hint that the sign change in the anomalous Hall signal is caused by a competition between bulk $\mathrm{Co}$ and interfacial contribution, with the spinpolarization of the interfacial $\mathrm{Pd}$ atoms possibly playing an important role for the latter.

Now, we come back to the temperature dependence of $\rho_{x y}$. Although temperature dependent calculations from first principles are challenging in general, the low temperature calculations could be qualitatively analyzed with respect to a potential temperature dependence in a crude approximation as follows: assuming a constant magnetization, the temperature dependence of the AHC is estimated by averaging it in the neighborhood of the Fermi energy $\mathrm{E}_{\mathrm{F}}$. Since the energy behavior of the side-jump contribution is rather smooth as compared to the intrinsic, it can be ignored for the estimate of the temperature dependence of the AHE. Thus, considering the intrinsic $\mathrm{AHC}$ in the $+/-0.03 \mathrm{eV}$ region around $\mathrm{E}_{\mathrm{F}}$, which roughly corresponds to $300 \mathrm{~K}$, the averaged $\mathrm{AHC}$ for $\mathrm{Co}_{1} \mathrm{Pd}_{8}$ would be always negative, while that for $\mathrm{Co}_{4} \mathrm{Pd}_{5}$ would be always positive for $\mathrm{T} \leq 300 \mathrm{~K}$. Nevertheless, for $\mathrm{Co}_{3} \mathrm{Pd}_{6}$ multilayers, the averaged $\mathrm{AHC}$ would decrease and could change its sign due to negative contributions for $|E| \geq 0.025 \mathrm{eV}$. This effect is not observed experimentally, however, as shown in Fig. 3(b), and the general trend is an increasing $\rho_{x y}$ with temperature. This means that for the temperature dependent considerations, it is not sufficient to take only the intrinsic and scattering-independent side-jump AHC into account by averaging it with respect to the energy and to ignore contributions to the AHE, which depend on the details of disorder at the interface and in the bulk. Although the individual temperature dependencies of the different contributions cannot be extracted from our data because of averaging over the whole sample, the limit of largest Co thickness shows that with increasing temperature the bulk at least gives rise to an increasing positive contribution in $\rho_{x y}$ in our $\{\mathrm{Co} / \mathrm{Pd}\}$ multilayers with thin Co layers and relatively large Pd thickness. Assuming that scattering at the interface is effectively a scattering at an impurity (in first order the temperature dependence of this scattering can be ignored) and taking into account that the bulk resistivity of Co (and Pd as well) increases with increasing temperature because of electron-phonon scattering or other inelastic excitations (e.g., magnons), it is reasonable to assume that the positive bulk contribution to $\rho_{x y}$ increases with temperature because of scattering dependent contributions to the AHE.

To summarize, we report on the Co thickness and temperature dependence of the Hall resistivity in $\left\{\mathrm{Co}^{0.20-0.55 \mathrm{~nm} /}\right.$ $\left.\mathrm{Pd}^{1.5 \mathrm{~nm} \text { or } 1.8 \mathrm{~nm}}\right\}_{9 \mathrm{x}}$ multilayer systems. In particular, a change of its sign was observed both in the dependence of $\rho_{x y}$ on the Co thickness and on the temperature. We have shown that the low temperature data can be understood on the base of calculations from first principles, which take only scatteringindependent contributions into account. Moreover, it has been shown that the simplest possible evaluation of the temperature dependence from these calculations is not enough to explain the experiments. However, taking the temperature dependence of $\rho_{x y}$ of the multilayers with largest Co thickness into account, it can be argued that the general increase of $\rho_{x y}$ with temperature is significantly influenced by the temperature dependence of the (positive) bulk contribution via phonons, magnons, etc. Furthermore, in the case of large Pd thickness investigated here, surface scattering seems to be not as important as in multilayer systems with significantly smaller Pd thickness as reported in Ref. 1.

We gratefully acknowledge Jülich Supercomputing Centre for computing time. V.K. and B.A. wish to thank for support by the Turkish State Planning Organization (Project No: 2009 K120730). J.S. and G.R. gratefully acknowledge financial support by the German Research Foundation DFG (Contract RE1052/24-1). J.W. was supported under Grant SPP1538 SpinCaT by the German Research Foundation DFG. H.Z., J.W., and Y.M. gratefully acknowledge funding by HGF-YIG Programme VH-NG-513.

${ }^{1}$ Z. B. Guo, W. B. Mi, R. O. Aboljadayel, B. Zhang, Q. Zhang, P. G. Barba, A. Manchon, and X. X. Zhang, Phys. Rev. B 86, 104433 (2012).

${ }^{2}$ E. H. Hall, Philos. Mag. 10, 301 (1880).

${ }^{3}$ P. F. Carcia, A. D. Meinhaldt, and A. Suna, Appl. Phys. Lett. 47, 178 (1985).

${ }^{4}$ S. Das, H. Yoshikawa, and S. Nakagawa, J. Appl. Phys. 93, 8098 (2003).

${ }^{5}$ H. S. Lee, S. B. Choe, S. C. Shin, and C. G. Kim, J. Magn. Magn. Mater. 239, 343 (2002).

${ }^{6}$ R. Sbiaa, H. Meng, and S. N. Piramanayam, Phys. Status Solidi RRL 5, 413 (2011).

${ }^{7}$ S. K. Wong, K. Srinivasan, R. Sbiaa, R. Law, E. L. Tan, and S. N. Piramanayagam, IEEE Trans. Magn. 46, 2409 (2010).

${ }^{8}$ L. Berger and G. Bergmann, in The Hall Effect and Its Applications, edited by C. L. Chien and C. R. Westgate (Plenum, New York, 1980), p. 56.

${ }^{9}$ E. M. Pugh, Phys. Rev. 36, 1503 (1930).

${ }^{10}$ S. Kim, S. R. Lee, and J. D. Chung, J. Appl. Phys. 73, 6344 (1993).

${ }^{11}$ S. U. Jen, B. L. Chao, and C. C. Liu, J. Appl. Phys. 76, 5782 (1994).

${ }^{12}$ Y. Aoki, K. Honda, H. Sato, Y. Kobayashi, S. Hashimoto, T. Yokoyama, and T. Hanyu, J. Magn. Magn. Mater. 162, 1 (1996).

${ }^{13}$ D. Rosenblatt, M. Karpovski, and A. Gerber, Appl. Phys. Lett. 96, 022512 (2010).

${ }^{14}$ N. Sato, J. Appl. Phys. 64, 6424 (1988).

${ }^{15}$ K. Fuchs, Proc. Cambridge Philos. Soc. 34, 100 (1938).

${ }^{16}$ G. Reiss, K. Kapfberger, G. Meier, J. Vancea, and H. Hoffmann, J. Phys.: Condens. Matter. 1, 1275 (1989).

${ }^{17}$ N. Nagaosa, J. Sinova, S. Onoda, A. h. MacDonald, and N. P. Ong, Rev. Mod. Phys. 82, 1539 (2010).

${ }^{18}$ S. Blügel and G. Bihlmayer, "Full-Potential Linearized Augmented Planewave Method," in Computational Nanoscience: Do It Yourself!, edited by J. Grotendorst, S. Blügel, and D. Marx, (John von Neumann Institute for Computing, Jülich, Germany, 2006), NIC Series, Vol. 31, ISBN 3-00017350-1, pp. 85-129, see https://www.flapw.de.

${ }^{19}$ J. Perdew, K. Burke, and M. Ernzerhof, Phys. Rev. Lett. 77, 3685 (1996). 
${ }^{20}$ A. Mostofi, J. R. Yates, Y.-S. Lee, I. Souza, D. Vanderbilt, and N. Marzari, Comput. Phys. Commun. 178, 685 (2008).

${ }^{21}$ F. Freimuth, Y. Mokrousov, D. Wortmann, S. Heinze, and S. Blügel, Phys. Rev. B 78, 035120 (2008).

${ }^{22}$ X. Wang, J. R. Yates, I. Souza, and D. Vanderbilt, Phys. Rev. B 74, 195118 (2006).
${ }^{23}$ A. A. Kovalev, J. Sinova, and Y. Tserkovnyak, Phys. Rev. Lett. 105, 036601 (2010).

${ }^{24}$ N. A. Sinitsyn, Q. Niu, J. Sinova, and K. Nomura, Phys. Rev. B 72, 045346 (2005).

${ }^{25}$ J. Weischenberg, F. Freimuth, J. Sinova, S. Blügel, and Y. Mokrousov, Phys. Rev. Lett. 107, 106601 (2011). 